\title{
Inter-beam Interference Reduction in Hybrid mmW Beamforming Transceivers
}

\author{
Muhammad Yasir Javed, Nuutti Tervo and Aarno Pärssinen \\ Centre for Wireless Communications (CWC), University of Oulu, Finland \\ \{yasir.javed, nuutti.tervo, aarno.parssinen\}@oulu.fi
}

\begin{abstract}
Practical radio frequency beamforming can suffer from high sidelobe levels which cause inter-beam interference (IBI) in multibeam transceivers. IBI can be reduced by shaping the amplitude and phase excitation over the individual antenna elements. However, such methods do not exploit the available power efficiently in practical arrays, where each antenna is driven with a dedicated power amplifier. In this paper, we show a simplified approach for hybrid beam synthesis in subarraybased beamforming architecture and propose a two-stage beamforming method for cancelling the IBI. The proposed technique cancels the interference between the subarrays while it maximizes the effective isotropic radiated powers in the desired directions. Simulation show typically over $40 \mathrm{~dB}$ IBI rejection for randomly spread beams and more than $4 \mathrm{~dB}$ improvement for radiated power compared to amplitude tapering.
\end{abstract}

\section{INTRODUCTION}

Recently announced third generation partnership project / new radio (3GPP/NR) specifications [1] verifies that the fifth generation $(5 \mathrm{G})$ wireless communication systems will employ millimeter-wave $(\mathrm{mmW})$ frequencies for the wide spectrum access. High frequencies enable also to pack multiple antennas in a relatively compact form factor and hence utilize the high antenna directivity to compensate for high path loss at $\mathrm{mmW}$ frequencies. Furthermore, multiple antennas enable the spatial domain interference management in the wireless systems. Due to the highly directional characteristic of mmW, line-off-sight (LOS) channel plays a dominant role in practical mmW systems [3], [4], [5]. However, utilizing directive communication requires steerable phased arrays which are capable of utilizing beamforming techniques over the antenna elements. Furthermore, 5G OFDM [1] waveforms with high-order modulations and extremely wide bandwidths require excellent error vector magnitude (EVM) in order to have decent bit error performance. High power consumption of wideband digital-to-analog converters (DACs) increases the power consumption of a fully digital beamforming approach, where each antenna has a dedicated DAC [2]. Correspondingly the same applies to analog-to-digital converters (ADCs) and digital baseband processing in the receiver. Therefore, RF/analog phased arrays are considered as a prior option for $\mathrm{mmW}$ beamforming systems. But for spatial separation of users, dedicated array is required for each beam. The beamforming can be also done in both analog and digital domains, where the former one is required for increasing the effective isotropic radiated power (EIRP) and the latter one is needed for spatial multiplexing of users or independent data-streams. Certainly, hybrid beamforming is the most energy-efficient way to allocate the array resources, it also forces to compromise between the directivity, the number of simultaneous beams, and power consumption of the transceiver.

The challenge in multi-beam transmitters is the interference between the beams. For maximizing the datarates for individual users, one needs to also maximize the signal-to-interference-plus-noise ratio (SINR) for each user. In standard radio frequency (RF) domain beam steering, the beams are always suffering from relatively large sidelobe level (SLL). Amplitude tapering (e.g. [6]) can be used to reduce the SLL, or Zero-forcing (ZF) [9], [8] can be used to reduce the interference in the desired directions. However, amplitude tapering or standard forms of $\mathrm{ZF}$ require changing the amplitude excitation over the antennas for shaping the beam. Changing the amplitude excitation from unitary limits the available EIRP, since the maximum power per RF branch is always limited by the the maximum output power of an individual power amplifier (PA) implemented either on-chip or as an external component.

Subarray-based systems allow utilizing multiple arrays to create notches to certain spatial directions [6], [7]. While each subarray can be used for creating the beams for individual users, they can be simultaneously used for interference cancellation without changing the amplitude excitation inside the subarray. In fact, such technique utilizes the actual RF beamformers for creating the notches in desired directions. In this paper, we propose a technique which cancels the inter-beam interference between multiple subarrays by cross-coupling the independent beams either in baseband, intermediate frequency (IF) or RF domain. The cross-coupling coefficients can be predefined based on the RF beamformers and known transmission directions. RF beamformers are used to maximize the power available from the subarray in a given direction, while the cancellation takes care of the interference between the beams. The 


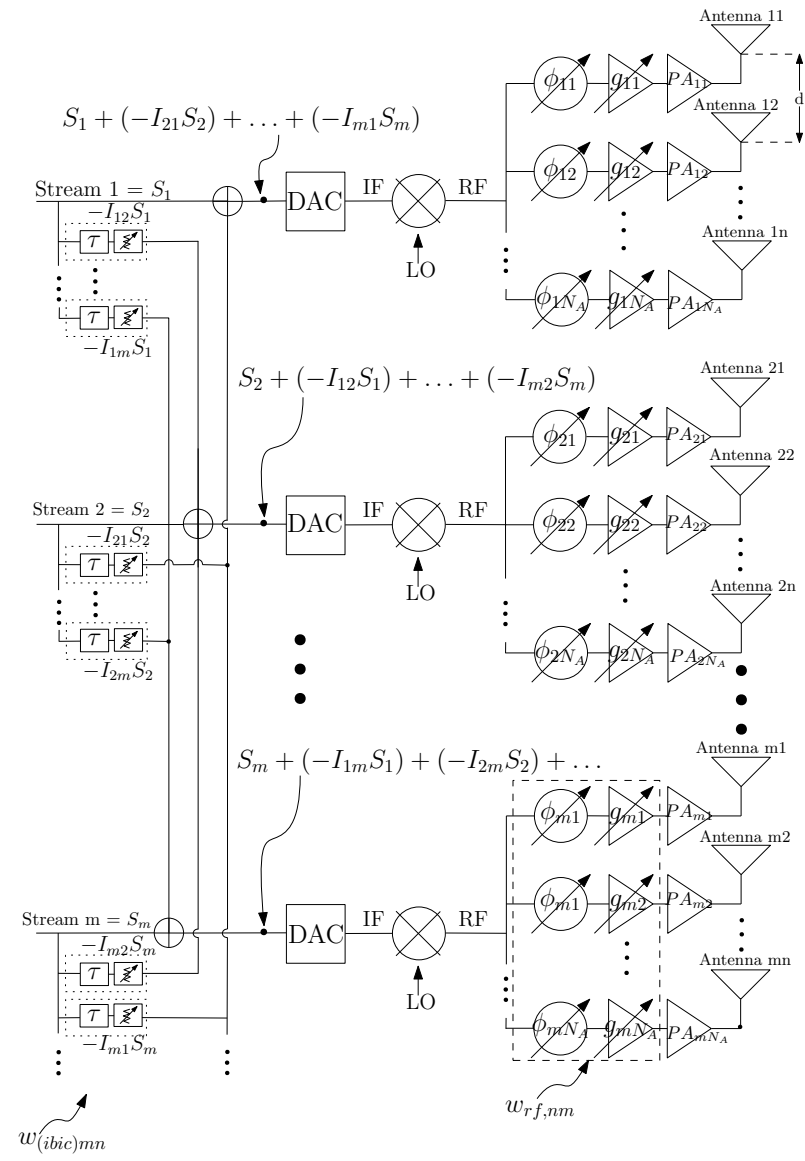

Fig. 1. Subarray based hybrid beamforming architecture.

proposed architecture and standard ways for cancelling IBI of multibeam transmitters are given in Sec. II. Sec. III gives statistical analysis of multibeam scenarios with the simulation results, followed by conclusion and discussion in Sec. IV.

\section{INTER-BEAM INTERFERENCE REDUCTION IN MULTI-BEAM Systems}

The system model for the subarray-based hybrid beamforming transmitter is presented in Fig. 1. In this architecture, each data-stream has a separate subarray equipped with $N_{A}$ antennas. The distance between adjacent antenna elements in subarrays is chosen to be $d=\lambda / 2$, where $\lambda$ is the wavelength of the carrier frequency $f_{0}$. IF, LO and RF signify intermediate frequency, local oscillator and radio frequency respectively. $\phi_{m n}$ and $g_{m n}$ denotes for phase shift and gain of $n^{t h}$ antenna of $m^{\text {th }}$ subarray, respectively. For the sake of simplicity, each antenna is assumed to be identically omnidirectional. In practice, patch antennas are typically used in antenna arrays and the impact of antenna element pattern can be included later straightforwardly. Each subarray is assumed to be a uniform linear array (ULA).

\section{A. Beam Steering in Subarrays}

Maximum ratio transmission (MRT) [10] is a coarse beamforming technique for maximizing the received power of each user. MRT for each subarray can be defined as [10]

$$
\mathbf{w}_{r f, m n}=\mathbf{h}_{m n}^{H}
$$

where $\mathbf{h}_{m n}$ is the channel vector from the $m$ th subarray to the $n$th user and ()$^{H}$ denotes the conjugate transpose of a vector. If we simply denote the channel of user $n$ as a unique spatial direction $\theta_{n}$ (i.e. line-of-sight (LOS) channel), the MRT refers to standard beam steering, which is done in RF domain in this example. For LOS channel, $\mathbf{h}_{m n}$ corresponds only a phase progression over the antenna elements and can be defined as [6]

$$
\begin{aligned}
\mathbf{h}_{m n}= & e^{j k(m-1) d \cos \left(\theta_{n}\right)} \\
& {\left[1, e^{j k d \cos \left(\theta_{n}\right)}, \ldots, e^{j k\left(N_{A}-1\right) d \cos \left(\theta_{n}\right)}\right]^{T}, }
\end{aligned}
$$

where $k=2 \pi / \lambda$ corresponds the wave number. The beam pattern of the $n$th subarray to the azimuth direction $\theta_{n}$ can be calculated by using array factor as [6]

$$
A F_{m}\left(\theta_{n}\right)=\mathbf{w}_{r f, m n} \mathbf{h}_{m n} .
$$

\section{B. Inter-beam Interference}

The antenna array beam pattern has side lobes around the main beam. In multibeam case, where different beams carry different data-streams, side lobes of one beam interfere with the main lobes of the other streams, causing inter-beam interference (IBI). IBI limits the achievable SINR at the receiver, i.e. it limits the achievable data-rate. IBI from the $m$ th to $n$th subarray can be calculated as

$$
I_{m n}=\frac{A F_{m}\left(\theta_{n}\right)}{A F_{n}\left(\theta_{n}\right)},
$$

where $A F_{m}\left(\theta_{n}\right)$ is the gain of $m$ th beam in the direction of $n$th user, and $A F_{n}\left(\theta_{n}\right)$ is the gain of $n$ beam in its own desired direction. The worst case IBI is around $12.3 \mathrm{~dB}$ (standard SLL of ULA) even if the main lobes are not overlapping [6]. Hence, without any interference reduction techniques, the beams are required to be relatively far from each other to achieve decent SINRs for the users. That will not be acceptable in densely populated $5 \mathrm{G}$ systems.

\section{Standard Ways of Reducing Inter-beam Interference}

IBI can be reduced e.g. by reducing the sidelobe level of the subarray beams or creating notches to the required directions by using ZF techniques. However, using such techniques in RF domain requires changing the amplitude excitation over the subarray elements. For an RF transmitter, this is challenging because of two reasons. Firstly, if the amplitude of each RF branch is controlled 


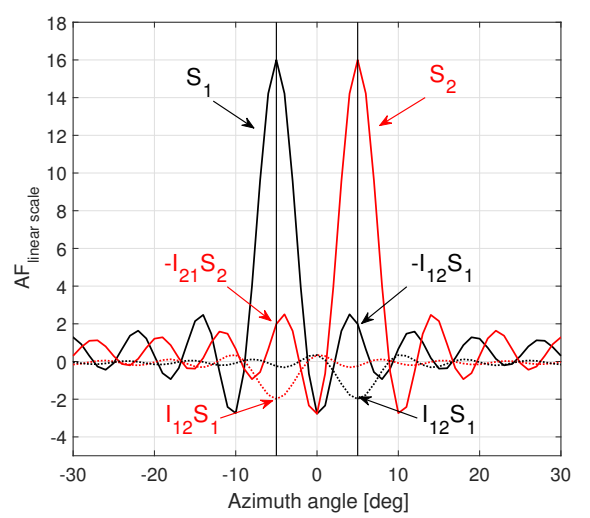

Fig. 2. Inter-beam interference cancellation signals in amplitude domain.

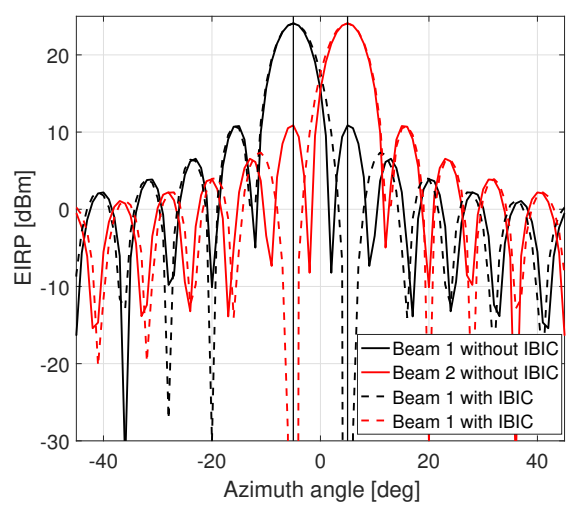

Fig. 3. Beams with and without IBIC.

for interference reduction, the parallel antenna-specific PAs experiences different shapes of nonlinearity which makes the array linearization challenging [11]. Secondly, the maximum power per RF branch is always limited by the PA technology. Hence, with unitary amplitude excitation, all PAs can be driven close to their limits. On the contrary, non-unitary amplitude distribution forces to scale the distribution according to the maximum achievable power level with the required linearity. In the receiver, similar problem occurs with noise versus receiver gain, if the amplitude levels are varied from unitary. This is because each analog signal combined in the combiner has individual signal level dependent SINR. In other words, changing amplitude distributions from unitary potentially reduces the achievable EIRP and linearity.

\section{Proposed Technique for Inter-beam Interference Cancellation}

An additional array can be used for creating a notch for the original beam to the desired direction. Such techniques have been presented already in [6]. However, using the additional array only for creating notches is a waste of antenna area. Moreover, if we consider that each user is equipped with a single subarray, we can use other subarrays to create the required notches for reducing the interference and simultaneously use them for beam steering of the other streams. Hence, each subarray should be cross-coupled to each other preferably in the digital domain in order to enable the ZF over the subarrays. In other words, we add a fraction of the interfering streams to the original beams in order to cancel the interference between the subarrays over-the-air. The described technique is called inter-beam interference cancellation (IBIC). The general idea is to add a scaled and phase-shifted (delayed) version of the interfering stream inside the beam under interference. The cross-coupling architecture was shown in Fig. 1. In two beam-scenario, the calculation of the cross-coupling coefficients can be simply calculated as $w_{(i b i c) m n}=-I_{m n}$. However, for a multi-beam cancellation, the cross-coupling coefficients may slightly change the original beams. Cross-coupling coefficients can be also defined based on the concept of the effective channel which is calculated over the beams. The effective channel over the subarray can be expressed as

$$
H_{\text {eff }}=\left(\begin{array}{cccc}
A F_{1}\left(\theta_{1}\right) & A F_{1}\left(\theta_{2}\right) & \ldots & A F_{1}\left(\theta_{N_{u}}\right) \\
A F_{2}\left(\theta_{1}\right) & A F_{2}\left(\theta_{2}\right) & \ldots & A F_{2}\left(\theta_{N_{u}}\right) \\
\vdots & \vdots & \ddots & \vdots \\
A F_{N_{u}}\left(\theta_{1}\right) & A F_{N_{u}}\left(\theta_{2}\right) & \ldots & A F_{N_{u}}\left(\theta_{N_{u}}\right)
\end{array}\right)
$$

where $N_{u}$ is the number of users and subarrays. Thus, the cross-coupling coefficients can be calculated as ZF coefficients defined for the effective channel as

$$
w_{i b i c}=H_{e f f}^{H}\left(H_{e f f} H_{e f f}^{H}\right)^{-1},
$$

where ()$^{-1}$ denotes the inverse of the matrix.

Transmitted interference cancellation signals combine destructively the interference from other steams in the direction of $\theta_{m}$ and cancel them over-the-air. Such an illustration is given in Fig. 2 with two beams in linear scale. It illustrates the interference in the directions of the beams and interference cancellation signals in those directions. Fig. 3 shows the corresponding beams with and without IBIC. The cancellation creates a notch for the beam 1 to the direction of beam 2 and vice versa, while the effect to the main lobe gain is negligible. Further away the beams are from each other, less impact the proposed technique has for the main lobe gain.

Comparison of the subarray power distributions with different interference reduction techniques and the corresponding beam patterns are shown in Fig 4. Taylor tapering with $-30 \mathrm{~dB}$ SLL target reduces all the sidelobes and hence it reduces the IBI. However, also EIRP is reduced because the antenna powers are required to be scaled according to the power limitations of the PAs. Because of the same reason, the EIRP in conventional ZF inside subarray is reduced compared to MRT. IBIC takes benefits from both MRT and ZF because it can 


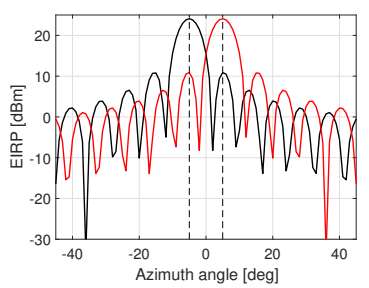

(a)

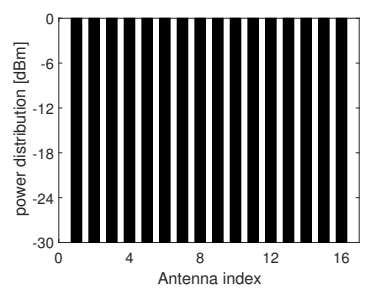

(e)

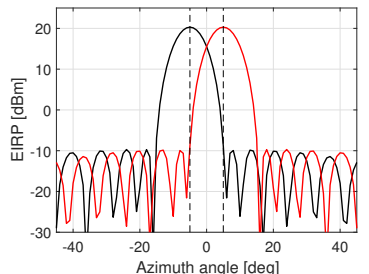

(b)

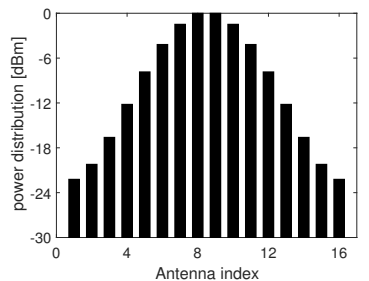

(f)

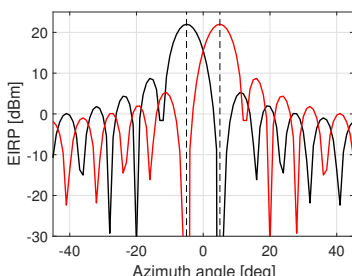

(c)

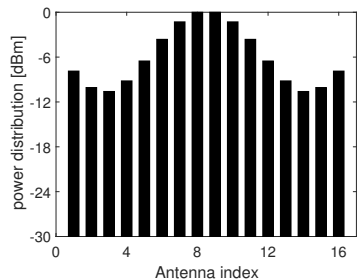

(g)

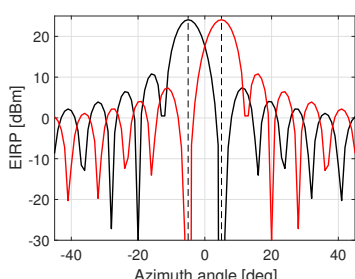

(d)

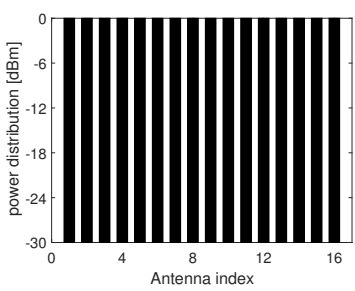

(h)

Fig. 4. Array beam patterns and power distributions over the subarray elements with (a) \& (e) MRT, (b) \& (f) Taylor, (c) \& (g) ZF, and (d) \& (h) IBIC, respectively

utilize unitary amplitude distribution over the array elements, while it forms the zeros in the desired directions by combining the signals from different subarrays.

\section{Statistical Analysis in Multi-BeAm SCENARIO}

Statistical simulations with dynamic beam configuration are performed in order to analyze the interference reduction techniques in a more realistic scenario. The antennas are divided into rows of 16 antennas, placed on top of each other. Each row is allocated to one sector by using MRT beamforming. Six beams are allocated for randomized directions within each sector. The simulation scenario is illustrated in Fig. 5 and Fig. 6. The subarrays are cross-coupled (as in Fig.1) in order to enable interference cancellation. In the simulations, we use 64-QAM modulated, $100 \mathrm{MHz}$ wide waveforms with raised cosine pulse shaping. The arrays are ULAs operating at $30 \mathrm{GHz}$ center frequency with identical omnidirectional patterns. The spacing between antenna elements in subarrays is chosen to be half of the wavelength at the operating frequency. Maximum power per PA having appropriate backoff for modulation is set to 0 $\mathrm{dBm}$. Number of simulated random beam configurations was 1000 .

SINRs are calculated for each stream by simulating the error vector magnitude (EVM) of the beams in their predefined directions. By using the EVM, the SINR can be simply written as SINR $=-20 \log _{10}(\mathrm{EVM})$. In this simulation, we assume that the EVM is only limited by the interference and pulse shaping filter (digital EVM).

Simulated probability density functions (PDFs) of EIRPs in the beamforming direction and SINRs over all the beams are shown in Fig. 7 and Fig. 8, respectively.
Taylor tapering with $-30 \mathrm{~dB}$ SLL, MRT, ZF inside the subarray and IBIC are compared. As expected, MRT maximizes the EIRP, but it limits the SINR close to the expected SLL. Amplitude tapering improves the SINR while it reduces the achievable EIRP because of scaled power distribution. ZF suppresses the interference even further and gives also decent performance in EIRP. However, in ZF EIRP value does has much more variance compared to the other techniques and the levels do not exceed MRT or IBIC. In fact, IBIC gives almost equivalent EIRP performance compared to MRT, while it has the same interference reduction performance than standard ZF. Hence, for simplified hybrid beamforming, IBIC allows to utilize the array resources efficiently for both power and interference reduction.

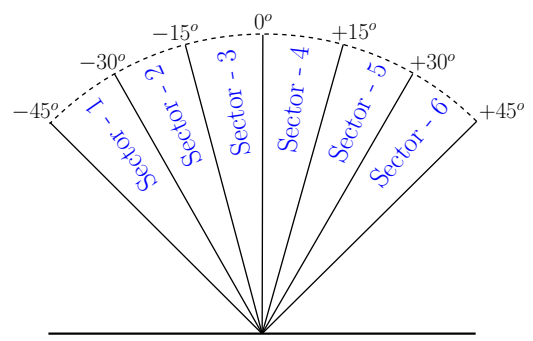

Fig. 5. Sectored simulation configuration.

\section{CONCLUSiOn AND Discussion}

Interference management is one of the key items when it comes to massive MIMO and $\mathrm{mmW}$ beamforming systems. Diverse techniques can be utilized to reduce the interference between beams. In this paper, we presented a technique which can cancel the interference in multi-beam transmitters by combining the signals 


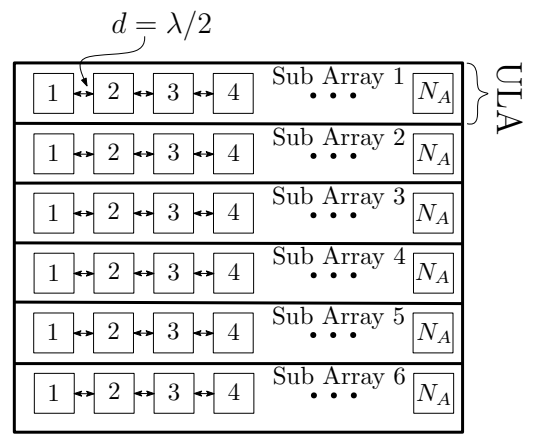

Fig. 6. Array configuration in the simulation.

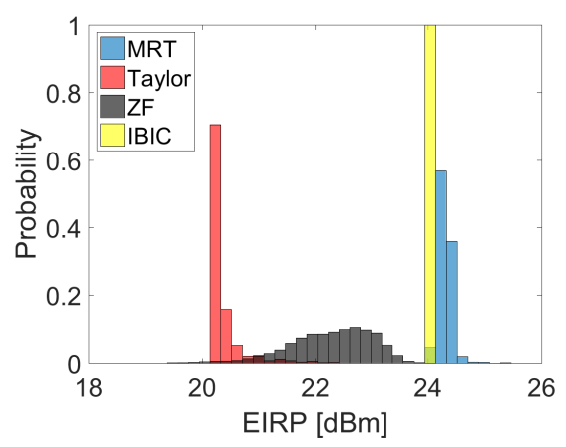

Fig. 7. PDFs of the simulated EIRP over the subarray beams.

from multiple subarrays over-the-air. Similar technique can be applied to receiver as well. It is concluded that the technique offers an RF implementation friendly approach for interference management due to the simplified RF beamforming schemes. Moreover, the actual RF beamformers are not required to be changed for the sake of interference reduction. Furthermore, we can even use unitary amplitude excitations, i.e. phase shifters only, in the RF, while the required notches can be designed in subarray basis. The proposed technique maximizes the EIRP in each beamforming direction, while it can significantly reduce the interference over the users. Typical SIR level of more than $40 \mathrm{~dB}$ in simulations with randomly spread directions of six beams exceed significantly performance achievable using amplitude tapering or conventional zero-forcing. In addition, EIRP compared to amplitude tapering can be more than $4 \mathrm{~dB}$ higher extending range significantly.

In practice, any beamforming technique is very sensitive for RF nonidealities such as nonlinearity of power amplifier [11], [12], errors in the phase shifters, antenna coupling, etc. Furthermore, beam squinting [6] of a wideband signal affects not only for beamforming direction but also for the directions of the notches. In baseband, the cancellation can be implemented in delay domain which can mitigate the bandwidth problem. Currently, the authors are working on an implementation of the technique and hence the future work will include

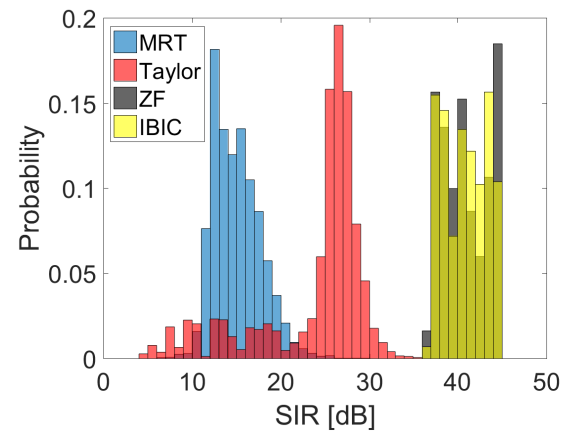

Fig. 8. PDFs of the simulated SIR over the subarray beams.

practical demonstrations with more detailed analysis on the effects of RF impairments.

\section{ACKNOWLEDGMENT}

This research has been financially supported by Nokia Corporation Ltd and Academy of Finland 6Genesis Flagship (grant 318927).

\section{REFERENCES}

[1] 3rd Generation Partnership Project; Technical Specification Group Radio Access Network; NR; Base Station (BS) radio transmission and reception(Rel 15). Available: http://www.3gpp.org/DynaReport/38-series.htm

[2] Shi, C. and Ismail, M. (2002). Data converters for wireless standards. Boston: Kluwer Academic Publishers.

[3] Y. H. Cho and J. J. Kim, "Line-of-Sight MIMO Channel in Millimeter-Wave Beamforming System: Modeling and Prototype Results," 2015 IEEE 81st Vehicular Technology Conference (VTC Spring), Glasgow, 2015, pp. 1-5.

[4] I. Sarris and A. R. Nix, "Design and Performance Assessment of High-Capacity MIMO Architectures in the Presence of a Line-of-Sight Component," in IEEE Transactions on Vehicular Technology, vol. 56, no. 4, pp. 2194-2202, July 2007.

[5] C. Sheldon M. Seo E. Torkildson M. Rodwell U. Madhow "Fourchannel spatial multiplexing over a millimeter-wave line-of-sight link" Microwave Symposium Digest 2009. MTT'09. IEEE MTT-S International. IEEE pp. 389-392 2009.

[6] Mailloux, R. (1994). Phased Array Antenna Handbook. ARTECH HOUSE.

[7] L. Zhang, A. Natarajan and H. Krishnaswamy, "Scalable Spatial Notch Suppression in Spatio-Spectral-Filtering MIMO Receiver Arrays for Digital Beamforming," in IEEE Journal of Solid-State Circuits, vol. 51, no. 12, pp. 3152-3166, Dec. 2016

[8] J. Noh, T. Kim, J. Y. Seol, and C. Lee, Zero-forcing based hybrid beamforming for multi-user millimeter wave systems, IET Communications, vol. 10, no. 18, pp. 26702677, 2016.

[9] M. Majidzadeh, A. Moilanen, N. Tervo, H. Pennanen, A. Tölli, M. Latva-aho. Partially Connected Hybrid Beamforming for Large Antenna Arrays in Multi-User MISO Systems. 28th Annual International Symposium on Personal, Indoor, and Mobile Radio Communications (PIMRC), Montreal, Canada, 2017

[10] T. K. Y. Lo, "Maximum ratio transmission" 1999 IEEE International Conference on Communications (Cat. No. 99CH36311), Vancouver, BC, 1999, pp. 1310-1314 vol.2.

[11] N. Tervo, J. Aikio, T. Tuovinen, T. Rahkonen and A. Pärssinen, "Effects of PA Nonlinearity and Dynamic Range in Spatially Multiplexed Precoded MIMO Systems", 22th European Wireless Conference, Oulu, Finland, 2016, pp. 1-6.

[12] Y. Zou, O. Raeesi, L. Antilla, A. Hakkarainen, J. Vieira, F. Tufvesson,Q. Cui, and M. Valkama, Impact of Power Amplifier Nonlinearities in Multi-User Massive MIMO Downlink, in IEEE Globecom Workshops 2015, Dec 2015, pp. 17. 